\title{
Lipoma digits: Commonest tumour - Uncommonest sites: Report of 2 cases
}

\author{
Ranjan Agrawal ${ }^{1, *}$, Miti Gupta ${ }^{2}$, Jagdamba Sharan ${ }^{3}$, Parbodh Kumar ${ }^{4}$
}

${ }^{1}$ Professor, Dept. of Pathology \& Surgery, Rohilkhand Medical College Hospital, Bareilly, Uttar Pradesh, India

\section{*Corresponding Author:}

Email: drranjan68@gmail.com

\begin{abstract}
Lipomas may occur anywhere within the body. However, their occurrence in the digits is very rare. We report 2 cases of lipoma in the digits. The first case is of a 70 years lady who had a soft painless swelling in the left thumb while the second case was of a 40 years lady who presented with a swelling over the medial aspect of right foot. Excision biopsy in both the cases proved them to be lipoma.

The cases are presented due to the rarity of their sites as only 14 cases of lipoma in the digits have been reported earlier.
\end{abstract}

Keywords: Lipoma, Digits.

\section{Introduction}

Lipomas of the tendon sheath of digits are very rare. The reported incidence is only $1 \% .^{1}$ The first case of lipoma of finger was reported by Stein in 1959. Approximately 14 cases of lipoma of fingers have been published in the literature so far. ${ }^{2}$ Lipomas, particularly of the digits have been reported only rarely. Lipomas may occur anywhere within the body. Because of their widespread distribution, there are a fair number of differential diagnoses.

Lipomas of foot and ankle are rare probably due to the relative paucity of soft tissues. ${ }^{3}$

\section{Case Reports}

Case 1: A 70 year female presented with a swelling over left thumb for 7 months. The swelling was sudden in onset and gradually progressive in nature. It was present both on the outer and inner surfaces. There was no associated pain. On examination the swelling was soft and boggy. FNAC showed mature fat cells suggestive of lipoma. Histopathology of the excision biopsy confirmed it to be lipoma (Fig $1 \& 2$ ). Surgical dissection and removal of the neoplasm was performed. No complications occurred during the postoperative period while the patient achieved full range of motion.

Case 2: A 40 year female presented with a swelling over medial aspect of right foot for one year. The swelling was of slow growing nature and non-painful. On examination, the swelling was globular and measured $1 \mathrm{~cm}$ in diameter and was present over the plantar aspect of right foot. It was soft, mobile and nontender. FNAC yielded greasy aspirate comprising of mature adipocytes suggestive of lipoma. Histopathology of the excised mass confirmed the diagnosis of lipoma (Fig 3). The patient is well even after 6 months of surgery with no evidence of recurrence thereafter.

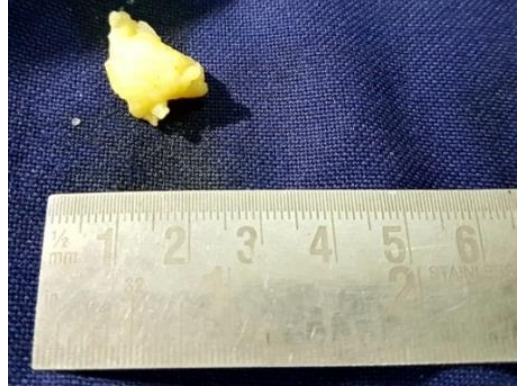

Fig.1: Showing gross appearance of tumour with glistening outer surface

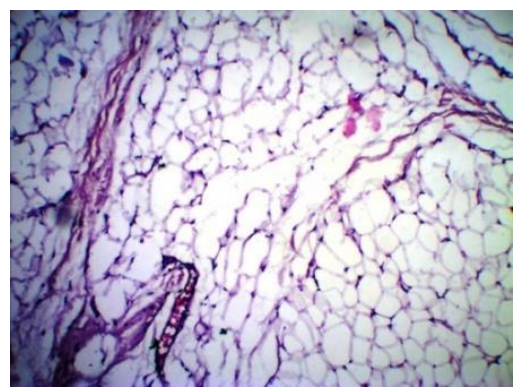

Fig. 2: Microscopic section of lipoma (H\&E x 400)

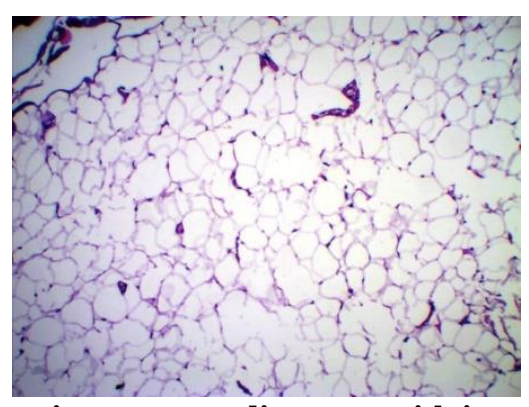

Fig 3: Showing mature adipocytes with interspersing thin walled blood vessels 


\section{Discussion}

Lipomas are benign neoplasms of mesenchymal origin occurring in areas where adipose tissue is abundant. They can occur anywhere in the body with approximately $15-20 \%$ located in the head and neck region followed by shoulder and back. ${ }^{4}$ They are rare in the hand and foot and those involving the digits are extremely rare, with only few cases reported. ${ }^{1}$

The first patient reported having lipoma of the finger was by Stein in 1959 and ever since only 14 cases are available in the literature. Majority of these cases had a preceding history of trauma. ${ }^{5}$

Lipomas are well encapsulated tumours and comprise of mature fatty tissue with a lipid droplet in the centre and the nucleus pushed to the periphery giving it a characteristic signet ring appearance. Lipomas account for more than 50\% of all soft-tissue tumours and $16 \%$ of soft-tissue mesenchymal tumours. ${ }^{6-8}$ Most common locations are in the upper back, neck, shoulder, chest and abdomen. ${ }^{9}$ However, they rarely present in the hand with a reported incidence of less than $1 \% .{ }^{10}$ They have been reported to occur both in the thenar or hypothenar areas. ${ }^{9}$ Both hands may be equally affected. Among the fingers, index and middle fingers are more commonly involved. Only a handful of cases involving the thumb are available. Lipomas occur most commonly in the fifth and sixth decade. ${ }^{2,7,11}$ They are more frequently seen in females and obese patients. ${ }^{12,13}$

The aetiology and pathogenesis of lipoma is still not well documented. Lipoblastic embryonic cell rests, fibroblasts undergoing fatty degeneration, metaplasia of the muscle cells, genetic, hormonal changes, trauma, chronic irritation, infections and/or infarction may be responsible for mature fat cells formation. ${ }^{8,13,14}$ Genetic theory proposes that spontaneous karyotypic anomalies lead to chromosomal fusion products causing proliferation of the fat cells. It is most commonly associated with translocations and rearrangements of the 12q13 q15 chromosomal region. Lipomas occurring after a trauma are due to herniation of adipocytes. Another hypothesis states that after blunt trauma certain growth factors, inflammatory cytokines and other mediators released by the damaged and necrotic cells induce the differentiation of preadipocytes to mature adipocytes. ${ }^{13,15}$ These have been termed pseudolipoma, since they do not have a fibrous capsule. Pseudolipomas occur mainly in the buttocks or thighs of females. ${ }^{15}$

Lipomas of the digits are often asymptomatic but may present as a solitary mass. ${ }^{2}$ Patients seek medical advice only when these lesions enlarge to cause mechanical impairment or are of cosmetic problem. ${ }^{2,8}$ Because of their enlarging size, they may lead to limitation of mobility and impaired grasping. Lateral deviation of the fingers may occur. They are slow growing, soft, fluctuant, bulging, lobulated and mobile masses. If associated with nerve compression, they may cause mild pain or distal sensory changes along with motor weakness. ${ }^{10}$

Radiological evaluation is diagnostic in up to $71 \%$ of cases. ${ }^{16}$ Computed tomography and especially magnetic resonance imaging are helpful in the assessment of such lesions and also in ruling out any malignant transformations. Histopathology remains the gold standard in the diagnosis of lipoma.

Differential diagnosis for a swelling in the hand is a large spectrum diseases encompassing both include neoplastic and nonneoplastic lesions. ${ }^{8,9,17}$ Lipoma on fingers is rarely considered clinically because of other common conditions such as ganglion cysts, giant cell tumours of tendon sheath, epidermal inclusion cysts, lipomas, neuromas, nerve sheath tumours, fibromas, glomus tumours, myxomas, angiolipomas and intraneural lipofibromas. Diagnosis in the hand and digits can be difficult, because of their rarity and deeper location. Lipoma of the foot should be differentiated from other lipomatous lesions such as fat herniation. ${ }^{16-}$ 18

Lipomas of the tendon sheaths and joints can be of two types. The first type occurs as solid fatty masses extending along the tendons for varying distances. They are termed as lipoma simplex symmetricum. They are less common and occur in the hand and wrist. When they occur in tendon sheath, these lesions are termed endovaginal tumours whereas deep lipomas arising outside the tendon sheath are known as epivaginal tumours. ${ }^{4}$ They may also arise from juxtra-articular regions or the periosteum (parosteal lipoma), sometimes causing nerve compression, erosion of bone or focal cortical hyperostosis. Patients generally present with mild aching pain in the digits or occasionally with trigger finger. The second type occurs as lipomatous lesions comprising of hypertrophic synovial villi distended with fat and are common in the knee joint. They are termed as lipoma arborescens.

Lipomas account for approximately $16 \%$ of all soft tissue mesenchymal tumours. ${ }^{12}$ World health organization (WHO) classifies benign lipomatous tumours into conventional lipoma, angiolipoma, fibrolipoma, spindle cell/pleomorphic lipoma, myxolipoma, chondroid lipoma, myolipoma, osteolipoma, lipomatosis, lipomatosis of nerve, lipoblastoma / lipoblastomatosis and hibernoma. Conventional lipomas are the most common types except in the parotid and lips where spindle cell lipomas are the commonest type. Secondary degenerative changes and atrophy are likely to occur and should not be mistaken with a malignant transformation.

Treatment depends mainly on the degree of functional impairment as well as on the cosmetic appearance. Best is simple surgical removal, irrespective of the histological subtype. Careful dissection is necessary so as to avoid recurrence. The 
reported recurrence rate is less than $5 \%$ within 1 year of resection. ${ }^{12}$ Malignant change is also very rare.

\section{Conclusion}

Although lipomas of the digits are rare entities, their awareness is important so as to differentiate them from other soft tissue tumours.

\section{References}

1. de Giorgi V, Salvini C, Sestini S, Alfaioli B, Carli P. Lipoma of the finger: A case report and differential diagnosis. Clin Exp Dermatol. 2005;30:439-40.

2. Chronopoulos E1, Nikolaos P, Karanikas C, Kalliakmanis A, Plessas S, Neofytou I et al. Patient presenting with lipoma of the index finger: a case report. Cases J. 2010 Jan 12;3:20. doi:10.1186/1757-1626-3-20.

3. Bakotic BW, Borkowski P: Primary soft-tissue neoplasms of the foot: the clinicopathologic features of 401 cases. J Foot Ankle Surg 2001;40:28-35.

4. Enzinger FM, Harvey DA. Spindle cell lipoma. Cancer 1975;36(5):1852-9. PMID:1192370.

5. Stein AH Jr. Benign neoplastic and nonneoplastic destructive lesions in the long bones of the hand. Surg Gynecol Obstet. 1959;109:189-97.

6. Kamra HT, Munde SL. "Lipoma on palmar aspect of thumb: a rare case report,” JCDR 2013;7 (8):1706-07.

7. Myhre-Jensen O: A consecutive 7-year series of 1331 benign soft tissue tumours. Clinicopathologic data. Comparison with sarcomas. Acta Orthop Scand 1981;52:287-93.

8. Ramirez-Montaño L, Lopez RP, Ortiz NS. "Giant lipoma of the third finger of the hand," Springer Plus 2013;2(1):164.

9. Nadar MM, Bartoli CR, Kasdan ML. Lipomas of the hand: a review and 13 patient case series. Eplasty. 2010 Oct 25;10:e66.

10. Ueno T, Ansai S, Matsuoka Y, Omi T, Kawana S. Lipoma Arising on the Sole of the Foot. The Internet Journal of Plastic Surgery. 2012;8 (1).

11. Rydholm A, Berg N: Size, site and clinical incidence of lipoma: factors in the differential diagnosis of lipoma and sarcoma. Acta Orthop Scand 1983; 54(6):929-934.

12. Ersozlu S, Ozgur AF, Tandogan RH: Lipoma of the index finger. Dermatol Surg 2007, 33:382-84.

13. Aust MC, Spies M, Kall S, Gohritz A, Boorboor P, Kolokythas P et al: Lipomas after blunt soft tissue trauma: are they real? Analysis of 31 cases. Br J Dermatol. 2007;157:92-99.

14. Akyol MU, Odzek A, Sokmensuer C. Lipoma of the tongue. Otolaryngol Head Neck Surg2000; 122:461-62.

15. Agrawal R. Lipoma tongue- a rare site for a common neoplasm. NJLM 2012; 1(1):43-44.

16. Signorini M, Campiglio GL: Posttraumatic lipomas: where do they come from? Plast Reconstr Surg 1998;101:699-705.

17. Gupta A, Singal R, Mittal A, Goyal M. Report of two cases of subcutaneous lipoma over the finger and review of literature: case series: benign tumour. Musculoskelet Surg. 2011;95(3):247-9.

18. Ozdemir HM, Yildiz Y, Yilmaz C, Saglik Y: Tumours of the foot and ankle: analysis of 196 cases. J Foot Ankle Surg 1997;36:403-8. 